\title{
ORIGINAL
}

\section{EXPOSICIÓN AL MONÓXIDO DE CARBONO DEL PERSONAL ESPECIALISTA EN EXTINCIÓN DE INCENDIOS FORESTALES}

\author{
Belén Carballo Leyenda (1), José A Rodríguez-Marroyo (2, 3), Jorge López-Satué (1), Concepción \\ Ávila Ordás $(2,3)$, Raúl Pernía Cubillo (1) y José Gerardo Villa Vicente $(2,3)$ \\ (1) Empresa de Transformación Agraria SA. (TRAGSA). \\ (2) Departamento de Educación Física y Deportiva, Universidad de León. \\ (3) Instituto de Biomedicina, Universidad de León.
}

\section{RESUMEN}

Fundamento: Se ha descrito que la salud y el rendimiento laboral del personal dedicado a la extinción de los incendios forestales se ven perjudicados principalmente por el monóxido de carbono $(\mathrm{CO})$. El objetivo de este trabajo ha sido analizar la exposición al $\mathrm{CO}$ a la que se ve sometido el personal especialista en la extinción de los incendios.

Métodos: Durante 58 incendios reales se monitorizó en 44 sujetos la exposición al $\mathrm{CO}$ a la que se vieron sometidos. También se analizó la concentración ponderada para una jornada de 8h (VA-ED). Todos los incendios fueron divididos en función del tipo de trabajo realizado (ataque directo, indirecto y combinado) y del combustible presente (pasto, matorral, bajo arbolado y mixto). Las variables analizadas fueron estudiadas mediante la prueba de Kruskal-Wallis, se usó el test de Mann-Whitney para establecer las diferencias entre medias.

Resultados: La concentración media de $\mathrm{CO}$ en los incendios fue de $18,4 \pm 1,7 \mathrm{ppm}$, lo que supuso una VA-ED de 7,0 $\pm 1,0 \mathrm{ppm}$. Las mayores exposiciones al CO se analizaron en los ataques combinados $(20,4 \pm 2,3 \mathrm{ppm})$ seguidas de las obtenidas en los ataques directos $(17,5 \pm 2,7 \mathrm{ppm})$ e indirectos $(10,6 \pm 5,4 \mathrm{ppm})$. Únicamente se obtuvieron diferencias significativas $(\mathrm{p}<0,05)$ al comparar las exposiciones al CO generadas por la combustión del matorral $(19,8 \pm 2,2)$ y bajo arbolado $(17,2 \pm 3,9)$ y pasto $(12,0 \pm 5,6)$.

Conclusiones: Las exposiciones de $\mathrm{CO}$ analizadas se vieron influidas por el tipo de combustible y trabajo desempeñado. Los valores medios obtenidos se situaron dentro de los límites establecidos como seguros por diferentes organismos nacionales (INSHT) e internacionales (NIOSH, OSHA).

Palabras clave: Monóxido de carbono. Incendio. Tipos de incendios. Incendios forestales. Exposición Profesional.

Correspondencia:

José A Rodríguez-Marroyo

Departamento de Educación Física y Deportiva.

Facultad de Ciencias de la Actividad Física y del Deporte, Universidad de León.

Campus de Vegazana s/n.

24071-León

j.marroyo@unileon.es

\section{ABSTRACT \\ Exposure to Carbon Monoxide in Wildland Firefighters during Wildfires Suppression}

Background: Health and occupational performance in wildland firefighters are mainly impaired for the carbon monoxide inhalation. Therefore, the aim of this study was to analyze the personal exposure to carbon monoxide in wildland firefighters during wildfires suppression.

Methods: Carbon monoxide exposure was monitorized in 44 subjects during 58 real wildfires. Moreover, we analyzed the time weighted average exposure for an $8 \mathrm{~h}$ shift (VA-ED). The wildfires were classified according to the work done (direct attack, indirect attack and mixed attack) and the current fuel (grass, bush, understory and mixed).

Results: The mean exposure to carbon monoxide was of $18,4 \pm 1,7 \mathrm{ppm}$, what supposed a VA-ED of $7,0 \pm 1,0 \mathrm{ppm}$. The highest exposures to carbon monoxide were found during the mixed attack $(20,4 \pm 2,3 \mathrm{ppm})$ and direct attack $(17,5 \pm 2,7 \mathrm{ppm})$. We only obtained significant differences $(\mathrm{p}<0.05)$ between bush $(19,8 \pm 2,2)$ and understory $(17,2 \pm 3,9)$ and grass $(12,0 \pm 5,6)$.

Conclusions: Exposures to carbon monoxide were influenced for the work done during the wildfires suppression and the type of fuel involved. Mean values obtained in this study were within safety limits described by different Spanish (INSHT) and international (NIOSH, OSHA) occupational safety and health agencies.

Key words: Carbon monoxide. Fire. Wildfire. Occupational exposure. Occupational health. Types of Fire.

Financiación. Este trabajo ha sido desarrollado por la Empresa de Transformación Agraria SA (TRAGSA), en colaboración con la Mutua Fraternidad Muprespa, el Departamento de Educación Física y Deportiva de la Universidad de León y la Empresa de Gestión Medioambiental SA (EGMASA) en el marco del proyecto de investigación «Factores condicionantes del rendimiento del personal especialista en extinción de incendios forestales», que ha contado con el apoyo del Área de Defensa Contra Incendios Forestales perteneciente a la Dirección General de Medio Natural y Política Forestal del Ministerio de Medio Ambiente y Medio Rural y Marino. 


\section{INTRODUCCIÓN}

En la actualidad, aproximadamente unas 35.000 personas se dedican a la extinción de incendios forestales en España aunque sea de forma temporal. El personal especialista en extinción de incendios forestales (PEEIF) se enfrenta durante la realización de su trabajo a condiciones desfavorables que influyen directamente en su rendimiento y en un aumento del riesgo laboral ${ }^{1}$. Se han descrito como factores condicionantes del rendimiento y la salud del PEEIF la exposición a altas temperaturas ${ }^{2}$, la inhalación de humos ${ }^{3-5}$, el trabajo con herramientas manuales en condiciones orográficas adversas $^{6}$, y la dificultad de rehidratación y avituallamiento ${ }^{7}$.

Diversos estudios a nivel internacional han analizado la exposición que el PEEIF experimenta a la mezcla de gases y partículas presentes en el humo, tanto en incendios forestales $^{3}$ como en quemas prescritas ${ }^{8-12}$. Todos ellos describen al monóxido de carbono $(\mathrm{CO})$ como el principal tóxico de interés durante la extinción de incendios forestales, sobre todo en exposiciones de alta concentración y corta duración, ya que su inhalación conlleva una serie de síntomas (mareos, nauseas, dolor de cabeza, irritación ocular y de las vías respiratorias, incremento de la frecuencia cardiaca y respiratoria, falta de concentración y disminución de las facultades cognitivas) que afectan tanto a la capacidad de los sujetos para realizar trabajo físico ${ }^{13,14}$ como a la toma de decisiones ${ }^{12}$. Además, las exposiciones a largo plazo pueden conllevar problemas cardiorrespiratorios que, conjugados con elevadas concentraciones, podrían derivar incluso en la muerte ${ }^{15,16}$. También los irritantes respiratorios (formaldehído y acroleína) ${ }^{11,18}$ y la fracción respirable de humo ${ }^{4}$ son considerados los principales elementos de estudio de la exposición al humo en incendios forestales.
La normativa española remite al Instituto Nacional de Seguridad e Higiene en el Trabajo (INSHT) como organismo capacitado para ofrecer los valores de referencia para la evaluación y control de los riesgos originados por la exposición de los trabajadores a agentes químicos, mediante la publicación anual de los Límites de Exposición Profesional para Agentes Químicos en España. La evaluación del riesgo por inhalación se lleva a cabo comparando la concentración medida en el aire ponderada en el tiempo, con el valor límite ambiental del agente en cuestión, tanto para exposiciones largas como de corta duración si el agente inhalado lo tuviera descrito ${ }^{18}$. Así, se definen tres tipos de exposiciones: i) exposición diaria al agente químico durante una jornada laboral estándar (8h), denominado valor ambiental de exposición diaria (VA-ED); ii) exposición de corta duración al agente químico para cualquier período de $15 \mathrm{~min}$ a lo largo de la jornada laboral, excepto para aquellos agentes químicos para los que se especifique un período de referencia inferior, denominado valor ambiental exposiciones cortas; iii) y valores límite para los agentes químicos que lo tienen asignado para la exposición diaria, pero no para períodos de corta duración (el CO en España). Actualmente no existe en ningún país un nivel de exposición específico para el PEEIF. Sin embargo, las guías de evaluación de la exposición que resultan más adecuadas son las del National Institute for Occupational Safety (NIOSH) y las de la American Conference of Governmental Industrial Hygienists (ACGIH) ${ }^{17}$. Los valores límite de exposición media para jornadas de $8 \mathrm{~h}$ tomados por el INSHT son idénticos a los referenciados por esta última institución (tabla 1).

Por todo ello, el objetivo de este trabajo ha sido analizar la exposición al $\mathrm{CO}$ a la que se ve sometido el PEEIF durante la extinción de incendios forestales, en función del tipo de trabajo realizado y del modelo de combustible implicado. 
Tabla 1

Valores límite de exposición para monóxido de carbono en partes por millón (ppm) en exposiciones promedio diarias para $8 \mathrm{~h}$ y en exposiciones de corta duración promedio para $15 \mathrm{~min}$ aportados por diferentes instituciones

\begin{tabular}{|c|c|c|c|}
\hline País & Estándar & $\begin{array}{c}\text { Exposición } \\
\text { diaria (8h) }\end{array}$ & $\begin{array}{c}\text { Exposición de } \\
\text { corta duración } \\
(\mathbf{1 5} \text { min) }\end{array}$ \\
\hline USA & OSHA (PELs) $^{\mathrm{a}}$ & 50 & \\
USA & NIOSH (RELs) & \\
USA & ACGIH (TLV) & 35 & $200^{\mathrm{g}}$ \\
Australia & NOSH (OES) $^{\mathrm{d}}$ & 25 & \\
Canadá & OHSR (PEVs) & 30 & 200 \\
España & INSHT (VLA) & 35 & 200 \\
\end{tabular}

${ }^{a}$ Occupational Safety and Health Administration (OSHA) Permissible Exposure Limits (PELs). ${ }^{\mathrm{b}}$ National Institute for Occupational Safety and Health (NIOSH) Recommended Exposure Limits (RELs). ${ }^{c}$ American Conference of Governmental Industrial Hygienists (ACGIH) Threshold Limit Values (TLVs). ${ }^{d}$ National Occupational Health and

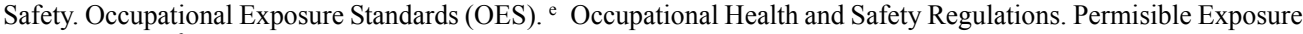
Values (PEVs). ${ }^{f}$ Instituto Nacional de Seguridad e Higiene en el Trabajo (INSHT) Valores Límite Ambientales (VLA). g Valor umbral que no debe ser sobrepasado en ningún momento durante la jornada ${ }^{18,29}$.

\section{SUJETOS Y MÉTODOS}

Sujetos. En el estudio participaron 44 sujetos (28 1 años, $175,3 \pm 0,8 \mathrm{~cm} \mathrm{y}$ $76,4 \pm 0,9 \mathrm{~kg}$ ) pertenecientes a las brigadas helitransportadas (BRIF) de Tabuyo del Monte (León), Pinofranqueado (Cáceres), Daroca (Zaragoza) y Puerto el Pico (Ávila). La experiencia profesional media de los sujetos fue aproximadamente de 3 años. Todos participaron voluntariamente y dieron su consentimiento por escrito. El estudio fue aprobado por el Comité de Ética de la Universidad de León y se realizó conforme a la Declaración de Helsinki para investigación con humanos.

Análisis del monóxido de carbono. La medición de la exposición al CO se llevó a cabo en 58 incendios reales durante las campañas de verano de los años 2007, 2008 y 2009. La concentración de CO se monitorizó de modo continuo cada 10 segundos mediante un dosímetro electrónico Tetra IV (Crowcon, Oxfordshire, UK), equipado con una sonda electroquímica por difusión y con un rango de medición de hasta 500 ppm. Los registradores fueron calibrados antes de cada incendio, mediante la opción de recalibrado y puesta a cero del propio instrumento. Además, una vez por semana fue calibrado mediante el uso de una sonda de calibración de $100 \mathrm{ppm}$ de CO. El dosímetro fue colocado en el tórax de 2 trabajadores en cada incendio. En todos los incendios se analizó la concentración media de $\mathrm{CO}$ y la concentración ponderada para una jornada de $8 \mathrm{~h}$ (VA-ED) en base a la metodología propuesta por el INSHT ${ }^{19}$ y la norma UNE-EN $689^{20}$.

$$
\mathrm{VA}-\mathrm{ED}=\mathrm{CO} \times \mathrm{T} \times 8^{-1}
$$

donde $\mathrm{CO}$ es la concentración de monóxido de carbono en ppm y T es el tiempo de análisis en $\mathrm{h}$.

Para determinar la influencia que tuvo el tipo de trabajo realizado en la exposición del PEEIF al CO se clasificó el trabajo en: ataque directo, ataque indirecto y ataque combinado. Se consideró como ataque directo al trabajo realizado directamente sobre la lla- 
ma con herramientas de sofocación (batefuegos, mochila extintora, motobomba,...). Se clasificó como ataque indirecto al trabajo realizado lejos del frente del incendio cuyo objetivo fue aislar el combustible de las llamas (construcción de líneas cortafuegos). El ataque directo combinado con fases de control y liquidación o remate (sofocar las zonas activas del incendio hasta lograr la extinción completa del fuego) se consideró como ataque combinado ${ }^{21}$. Por último, los incendios se clasificaron en función del combustible más representativo sobre el que se actuó. Basándonos en la clasificación de 13 modelos establecida por Rothermel ${ }^{22}$ el combustible se clasificó en: pasto, matorral, bajo arbolado y mixto (combinación de los anteriores).

Análisis estadístico. Los resultados se expresan como media \pm error estándar de la media (EEM) y media geométrica \pm desviación estándar geométrica (DEG). Las variables analizadas fueron estudiadas mediante la prueba de Kruskal-Wallis, se usó el test de Mann-Whitney para establecer las diferencias entre medias. Fueron considerados estadísticamente significativos los valores de $p<0,05$. Para el análisis estadístico se usó el software SPSS+ vers. 15.0 statistical software (Chicago, IL).

\section{RESULTADOS}

En la tabla 2 se muestra la distribución del tipo de combustible y trabajo realizado en los incendios analizados. Las concentraciones de $\mathrm{CO}$ analizadas en los incendios se muestran en la tabla 3. Se observó una gran variabilidad entre los valores alcanzados en los diferentes incendios, obteniéndose un rango entre 0,7-57,8 ppm. Cuando se calcularon los valores de CO para jornadas de $8 \mathrm{~h}$ los valores obtenidos fueron menores.

Las concentraciones de $\mathrm{CO}$ a las que se vio sometido el PEEIF estuvieron influidas por el tipo de trabajo realizado (tabla 4). Las mayores concentraciones se obtuvieron en el ataque combinado $(20,4 \pm 2,3)$, encontrándose diferencias significativas $(p<0,05)$ entre los valores analizados en el ataque combinado y ataque indirecto. Sin embargo, cuando se analizó VA-ED únicamente se obtuvieron diferencias significativas $(p<0,05)$ entre las concentraciones de $\mathrm{CO}$ analizadas en el ataque combinado y ataque directo. Posiblemente debido a la menor duración $(\mathrm{p}<0,05)$ de la exposición cuando se realizó el ataque directo $(102,9 \pm 18,7$ vs. $202,9 \pm 21,3 \mathrm{~min})$.

Los resultados obtenidos en función del tipo de combustible se muestran en la Tabla 5 . Se obtuvieron diferencias significati-

Tabla 2

Distribución (\%) del tipo combustible y trabajo realizado en los incendios analizados

\begin{tabular}{|l|c|c|c|c|}
\hline \multicolumn{1}{|c|}{ País } & $\begin{array}{c}\text { Ataque } \\
\text { Directo }\end{array}$ & $\begin{array}{c}\text { Ataque } \\
\text { Indirecto }\end{array}$ & $\begin{array}{c}\text { Ataque } \\
\text { Combinado }^{\mathbf{a}}\end{array}$ & Total \\
\hline Pasto & 18,7 & 14,2 & & 6,9 \\
Matorral & 62,5 & 42,9 & 74,3 & 67,2 \\
Bajo arbolado & 18,8 & 42,9 & 17,1 & 20,7 \\
Mixto & & & 8,6 & 5,2 \\
Total & 27,6 & 12,1 & 60,3 & \\
\hline
\end{tabular}

a Se ha considerado como ataque combinado al ataque directo realizado junto a labores de remate y/o liquidación. 
Tabla 3

Datos globales para la exposición del PEEIF al monóxido de carbono en la extinción de incendios forestales analizados durante las campañas 2007, 2008 y 2009

\begin{tabular}{|l|c|c|c|}
\hline & $\begin{array}{c}\text { Media } \\
\text { aritmética } \\
( \pm \mathbf{E E M})\end{array}$ & $\begin{array}{c}\text { Media } \\
\text { geométrica } \\
( \pm \mathbf{D E G})\end{array}$ & Rango \\
\hline CO (ppm) & $18,4 \pm 1,7$ & $13,4 \pm 2,5$ & $0,7-57,8$ \\
VA-ED (ppm) & $7,0 \pm 1,0$ & $3,7 \pm 3,6$ & $0,1-35,7$ \\
Duración (min) & $173,4 \pm 16,8$ & & $22-540$ \\
\hline
\end{tabular}

CO: concentración media de monóxido de carbono. VA-ED: exposición promedio al CO relativizada para jornadas de 8 h.

Tabla 4

Valores medios ( \pm EEM) de exposición al monóxido de carbono durante la extinción de incendios forestales en función del tipo de ataque

\begin{tabular}{|l|c|c|c|}
\hline & Directo & Indirecto & Combinado \\
\hline CO (ppm) & $17,5 \pm 2,7$ & $10,6 \pm 5,4^{*}$ & $20,4 \pm 2,3$ \\
VA-ED (ppm) & $3,9 \pm 1,0^{*}$ & $7,0 \pm 4,9$ & $8,4 \pm 1,4$ \\
Duración (min) & $102,9 \pm 18,7^{*}$ & $186,7 \pm 68,8$ & $202,9 \pm 21,3$ \\
\hline
\end{tabular}

CO: concentración media de monóxido de carbono. VA-ED: exposición promedio al CO relativizada para jornadas de 8 h. *, diferencias significativas con el ataque combinado $(\mathrm{p}<0,05)$.

Tabla 5

Valores medios ( \pm EEM) de exposición al monóxido de carbono durante la extinción de incendios en función del tipo de combustible

\begin{tabular}{|l|c|c|c|c|}
\hline & Mixto & Pasto & Matorral & Bajo arbolado \\
\hline CO (ppm) & $13,8 \pm 4,6$ & $12,0 \pm 5,6$ & $19,8 \pm 2,2^{*}$ & $17,2 \pm 3,9^{*}$ \\
VA-ED (ppm) & $5,8 \pm 1,2^{*}$ & $0,9 \pm 0,4$ & $7,8 \pm 1,3^{*}$ & $6,6 \pm 2,8^{*}$ \\
Duración (min) & $225,3 \pm 65,0^{*}$ & $36,0 \pm 5,1$ & $187,2 \pm 20,7^{*}$ & $161,3 \pm 37,2^{*}$ \\
\hline
\end{tabular}

CO: concentración media de monóxido de carbono. VA-ED: exposición promedio al CO relativizada para jornadas de 8 h. * , diferencias significativas con Pasto $(\mathrm{p}<0,05)$.

vas $(\mathrm{p}<0,05)$ al comparar la concentración de CO analizadas en los incendios cuyo combustible fue el matorral y el bajo arbolado con los incendios de pasto. Las meno- res $(\mathrm{p}<0,05)$ concentraciones de VA-ED $(0,9 \pm 0,4)$ y duraciones de trabajo $(36,0 \pm 5,1)$ se encontraron en los incendios de pasto. 


\section{DISCUSIÓN}

A pesar de que existen diferentes estudios que han analizado la exposición al $\mathrm{CO}$ y otros tóxicos en bomberos forestales estadounidenses ${ }^{3,8-10}$ no existen estudios en nuestro país que analicen la exposición al humo que soporta el PEEIF durante el desempeño de su trabajo. Por ello, los resultados obtenidos representan la primera aproximación que caracteriza la exposición al $\mathrm{CO}$ del PEEIF en España. Nuestros resultados muestran un nivel de exposición al $\mathrm{CO}$ durante $173,4 \pm 16,8 \mathrm{~min}$ de $18,4 \pm 1,7 \mathrm{y}$ $13,4 \pm 2,5$ ppm para la media aritmética y geométrica, respectivamente. Estos resultados difieren de los obtenidos por otros autores en bomberos forestales. Reh y cols. ${ }^{9}$ analizaron exposiciones al $\mathrm{CO}$ de $\sim 18 \mathrm{ppm}$ (media geométrica). Sin embargo, Reinhardt y Ottmar ${ }^{3}$ y McCammon y McKenzie ${ }^{10}$ analizaron exposiciones de $\sim 7,5 \mathrm{ppm}$ (media geométrica) durante $\sim 200 \mathrm{~min}$ y $\sim 3 \mathrm{ppm}$ (media aritmética) durante $\sim 400 \mathrm{~min}$, respectivamente. Posiblemente estas diferencias sean debidas a la diferente composición vegetal del combustible (composición específica, densidad y humedad,...), ya que éste es el principal factor que condiciona la composición del humo ${ }^{10}$. Así, en este estudio las mayores concentraciones de $\mathrm{CO}$ se alcanzaron cuando el combustible fue de tipo matorral $(19,8 \pm 2,2)$. Estos resultados son similares a los encontrados por Reinhardt ${ }^{23}$ en quemas experimentales ( $22 \mathrm{ppm})$. Sin embargo, Reisen y cols. ${ }^{12}$ en quemas experimentales con diferentes combustibles analizaron valores de $\sim 13$ ppm para el matorral, aunque de todos los combustibles estudiados éste provocó las mayores concentraciones de $\mathrm{CO}$. Las exposiciones al $\mathrm{CO}$ a las que se sometió el PEEIF fueron inferiores a las referenciadas en la literatura ${ }^{24,25}$ para bomberos de estructura (12-1.087 ppm), debido a la mayor concentración de gases nocivos presentes en la combustión de los elementos estructurales de los edificios y a la realización del trabajo en espacios confinados ${ }^{26}$.
Otro factor que pudo afectar a la exposición al CO de los sujetos que participaron en este estudio fue el tipo de trabajo realizado en los incendios. Éste va a depender de diversos factores como el tipo de combustible, la orografía del terreno y las condiciones meteorológicas y va a condicionar tanto la distancia que existe entre el bombero y la fuente de $\mathrm{CO}$ como el tiempo de exposición ${ }^{5}$. Comúnmente el trabajo realizado en los incendios se clasifica en ataque directo o indirecto, en función de la distancia que existe entre el trabajador y la llama ${ }^{27}$. Debido a que en muchos de los incendios analizados $(\sim 60 \%)$ además de realizar un ataque directo también se ejecutaron tareas de control y remate, se procedió a clasificar este tipo de trabajo como ataque combinado. Las menores exposiciones al $\mathrm{CO}$ se registraron en el ataque indirecto, ya que el PEEIF trabajó a una mayor distancia de la llama. Este tipo de trabajo se caracteriza por la construcción de líneas de defensa (pequeñas áreas cortafuegos) para evitar el avance de las llamas $^{27}$. Por ello, el PEEIF trabajó a una mayor distancia de la llama, por lo que la exposición al humo fue menor. Sin embargo, en el ataque directo los bomberos se ven sometidos a una mayor exposición al humo ${ }^{28}$ y por lo tanto a una mayor concentración de $\mathrm{CO}$. Contrariamente a lo que se pudiera pensar, fue en el ataque combinado donde los sujetos de este estudio se vieron expuestos a las mayores concentraciones de CO. Las labores de control y liquidación del fuego son características de este tipo de ataque y su objetivo es la extinción completa del fuego. En este tipo de tareas el PEEIF controla y sofoca las posibles reproducciones del fuego producidas por los rescoldos existentes. Esta circunstancia genera una mayor producción de humo y CO debido a la combustión incompleta de la biomasa ${ }^{28}$. Nuestros datos son similares a los obtenidos por Reinhardt y $\mathrm{Ottmar}^{3}$ en quemas prescritas para ataques indirectos ( $12 \mathrm{ppm}$ ) y combinados (ataque directo más remate del incendio) ( 21 ppm). Sin embargo, los resultados analizados por estos autores durante la realización del ata- 
que directo ( $\sim 33 \mathrm{ppm})$ fueron mayores a los descritos en este trabajo, posiblemente debido a la mayor duración de los incendios y al tipo de combustible implicado. El estudio de Reinhardt y Ottmar ${ }^{3}$ se llevó a cabo en Estados Unidos, en una zona caracterizada por la presencia de combustibles gruesos y abundancia de combustible seco en el suelo, que se asocian con elevadas exposiciones al $\mathrm{CO}^{17}$. Por el contrario, en el presente trabajo el principal combustible fue el matorral.

La concentración media de $\mathrm{CO}$ analizada en este trabajo supondría, para una jornada laboral de $8 \mathrm{~h}$, una exposición de 7,0 $0 \pm 1,0$ ppm. Este valor se encuentra dentro del rango de seguridad (hasta $25 \mathrm{ppm}$ ) establecido por el INSHT ${ }^{18}$ para exposiciones laborales en España y no superaría tampoco los límites establecidos por organismos internacionales como la $\mathrm{NIOSH}^{29}(35 \mathrm{ppm})$ y la $\mathrm{OSHA}^{30}(50$ ppm). A pesar de los bajos valores estudiados, debe tenerse en cuenta que el VA-ED se ve influido por la corta duración de las exposiciones. Este hecho provoca que para su cálculo tenga más relevancia la duración de la exposición que la concentración de CO. Por ello, se ha afirmado que su uso no resulta adecuado para cuantificar las exposiciones altamente intermitentes (condiciones meteorológicas cambiantes, variaciones en la tasa de emisión de CO generado en la combustión) que tienen lugar en los incendios forestales ${ }^{4}$. Parece más adecuado analizar este tipo de exposición en periodos de corta duración y comparar los resultados con los valores límite ambientales establecidos para dicho período ${ }^{10}$. En este sentido, en nuestro país se establece la concentración de 75 ppm como límite de exposición al CO para un periodo de $30 \mathrm{~min}^{18}$. Otros autores proponen la utilización de factores de corrección del límite de exposición ambiental en jornadas laborales que no cumplen el estándar ${ }^{4,31,32}$.

En conclusión las concentraciones medias de CO obtenidas en los incendios forestales analizados se vieron influidas por el tipo de combustible y el tipo de trabajo realizado. Los mayores valores se obtuvieron cuando el combustible quemado fue el matorral y se realizó un ataque combinado. Los valores medios analizados se situaron dentro de los límites establecidos como seguros por diferentes organismos nacionales (INSHT) e internacionales (NIOSH, OSHA). Sin embargo, debido a la duración de los incendios estudiados en este trabajo, el cálculo del valor límite ambiental de exposición diaria para jornadas de 8h (VA-ED) parece no ser recomendable, ya que puede infravalorar la exposición real al CO. Por ello, futuros trabajos deberían analizar en cortos periodos de tiempo el nivel de exposición al que se ve sometido el PEEIF durante la extinción de los incendios.

\section{BIBLIOGRAFÍA}

1. Aisbett B, Nichols B. Fighting fatigue whilst fighting bushfire: an overview of factors contributing to firefighter fatigue during bushfire suppressions. Aust J Emergen Manage. 2007; 22:31-39.

2. Rossi R. Fire fighting and its influence on the body. Ergonomics. 2003; 46:1017-1033.

3. Reinhardt TE, Ottmar RD. Baseline measurements of smoke exposure among wildland firefighters. J Occup Environ Hyg. 2004; 1:593-606.

4. Austin CC. Wildland firefighter health risks and respiratory protection. The Robert- Sauvé Institute for Research in Occupational Health and Safety of Quebec (Institut de recherche Robert-Sauvé en santé et en sécurité du travail (IRSST). Montreal: 2008. Report R-572. Disponible en: htpp://www. irsst.qc.ca.

5. Reisen F, Brown SK. Australian firefighters' exposure levels to air toxics during bushfire burn of autumn 2005 and 2006. Environ Inter. 2009; 35:342-352.

6. Apud E, Gutierrez M, Lagos S, Maureira F, Meyer F, Espinoza J. Manual de ergonomía forestal. Chile: Valverde; 1999.

7. Espinosa V, Roca E, Drobnic F, Prat R, Mirallas J, Barbany JR. Suplementación con glicerina para prevenir la deshidratación en tareas simuladas de bomberos. Arch Med Deporte. 2007; 120:253-61. 
8. Materna BL, Jones JR, Sutton PM, Rothman N, Harrison RJ. Occupational Exposures in California Wildland Fire Fighting. Am Ind Hyg Assoc J. 1992; 53:69-76.

9. Reh CM, Letts D, Deitchman S. Health hazard evaluation report. U.S. Department of the Interior National Park Service, Yosemite National Park. California: U.S National Institute of Occupational Health and Safety (NIOSH); 1994.

10. McCammon JB, McKenzie L. Wildland firefighter managed carbon monoxide (CO) exposure monitoring. March (CO): U.S. National Institute for Occupational Safety and Health (NIOSH); 2000.

11. Reinhardt TE, Ottmar RD, Hanneman A. Smoke exposure among firefighters at prescribed burns in the Pacific Northwest. United States Departement of Agriculture Forest Service. Pacific Northwest Research Station. Research Paper 526. 2000. p. U145.

12. Reisen F, Brown S, Cheng M. Air Toxics in Bushfire Smoke: Firefighter's Exposure during Prescribed Burns. Coimbra: 5th International Conference on Forest Fire Research; 2006.

13. Austin CC. Accurate solution of the Coburn-Forster-Kane (CFK) equation using an iterative algorithm programmed into Excel spreadsheet software. New Orleans: American Industrial Hygiene Association Conference and Exhibition; 2001.

14. Scarino A, Tardif R. Modélisation de l'exposition au monoxyde de carbone. Studies and Research Reports. Montreal: Institut de Robert-Sauvé en santé et sécurité du travail (IRSST); 2005.

15. Raub J, Mathieu-Nolf M, Hampson N, Thom S. Carbon monoxide poisoning: a public health perspective. Toxicology. 2000; 145:1-14.

16. Carlisle AJ, Sharp NCC. Exercise and outdoor ambient air pollution. Brit J Sport Med. 2001; $35: 214-222$.

17. Reinhardt TE, Ottmar RD. Smoke exposure among wildland firefighters a review and discussion of current literature. United States Department of Agriculture Forest Service. Pacific Northwest Research Station. Gen Tech Rep. PNW-GTR-373. 1997.

18. Instituto Nacional de Seguridad e Higiene en el Trabajo (INSHT). Límites de exposición profesional para agentes químicos en España [monografía en Internet]. Madrid: INSHT; 2010. Disponible en: http://www.insht.es/InshtWeb/Contenidos/Docu-
mentacion/TextosOnline/Valores_Limite/Limites 2010/LEP\%202010\%20V.Marzo\%202010 $\% 283$ marzo $\% 29$.pdf.

19. Instituto Nacional de Seguridad e Higiene en el Trabajo (INSHT). Guía técnica para la evaluación y prevención de los riesgos presentes en el trabajo relacionados con agentes químicos [monografía en Internet]. Madrid: INSHT; 2003. Disponible en: http://www.insht.es/InshtWeb/Contenidos/Normativa/GuiasTecnicas/Ficheros/g_AQ.pdf

20. UNE-EN 689. Atmósferas en el lugar de trabajo. Directrices para la evaluación de la exposición por inhalación de agentes químicos para la comparación con los valores límite y estrategia de la medición. Madrid: Agencia Española de Normalización; 1996.

21. Martínez-Ruíz, E. Organización del combate en incendios forestales. En: Vélez R (coodinador). La defensa contra incendios forestales: Fundamentos y experiencias. Madrid: McGraw-Hill Interamericana; 2000. p.21.1-21.5.

22. Rothermel RC. How to predict the spread and intensity of forest and range fires. United States Department of Agriculture Forest Service. Intermountain Forest and Range Experimentation. Paper 143. 1983.

23. Reinhardt TE. Firefighter smoke exposure at prescribed burns a study and action recommendation [Unpublished report on file with U S Department of Agriculture], United States Department of Agriculture Forest Service. Pacific Northwest Research Station, Fire and Air Resource Management Project, 4043 Roosevelt Way NE Seattle, WA 981056497; 1989.

24. Brandt-Rauf PW, Fallon LF, Tarantini T, Idema C, Andrews L. Health hazards of fire fighters: exposure assessment. British J Ind Med. 1988; 45:606612.

25. Boldstad-Johnson DM, Burgess JL, Crutchfield CD, Storment S, Gerkin R, Wilson JR. Characterization of firefighter exposures during fire overhaul. AIHAJ. 2000; 61:636-641.

26. Treitman RD, Burgess WA, Gold A. Air contaminants encountered by firefighters. Am. Ind. Hyg Assoc J. 1980; 41:796-802.

27. Budd GM, Brotherhood JR, Hendrie AL, Jeffery SE, Beasley FA, Costin BP, et al. Project Aquarius 4. Experimental Bushfires, Suppression Procedures, and Measurements. Int J Wildland Fire. 1997; 7:99-104. 
28. Alvarado C, Pascual I. Riesgos físicos no comunes en la extinción de incendios forestales y su prevención con EPI adecuados. $4^{\mathrm{a}}$ Conferencia Internacional sobre Incendios Forestales. Sevilla; 2007. Disponible en: http://www.fire.uni-freiburg.de/sevilla2007/contributions/doc/cd/SESIONES_TEMATICAS/ST6/Alvarado_Pascual_SPAIN_DGB.pdf.

29. National Institute for Occupational Safety and Health (NIOSH). Pocket guide to chemical hazards. Cincinnati: US National Institute for Occupational Safety and Health (NIOSH); 2005.
30. Occupational Safety and Health Administration (OSHA). Permissible exposure limites (PELs). Washington:U.S. Occupational Safety and Health Administration; 2003.

31. Brief R, Scala R. Occupational Exposure Limits for Novel Work Schedules. Am Ind Hyg Assoc J. 1975; 36:467-469.

32. Brodeur J, Vyskocil A, Tardif R, Perrault G, Drolet D, Truchon G, Lemay F. Adjustment of permissible exposure values to unusual work schedules. Am Ind Hyg Assoc J. 2001; 62:584-594. 